\title{
Special issue: Dynamics of systems on the nanoscale (2018) Editorial $^{\star}$
}

\author{
Ilko Bald ${ }^{1}$, Ilia A. Solov'yov ${ }^{2}$,, Nigel Mason $^{3}$, and Andrey V. Solov'yov ${ }^{4,5}$ \\ 1 Institute of Chemistry, University of Potsdam, Karl-Liebknecht-Str. 24-25, 14476 Potsdam, Germany \\ 2 Department of Physics, Carl von Ossietzky Universität Oldenburg, Carl-von-Ossietzky-Straße 9-11, \\ 26129 Oldenburg, Germany \\ 3 School of Physical Sciences, The University of Kent Canterbury, CT2 7NH Canterbury, UK \\ 4 MBN Research Center gGmbH, Altenhoferallee 3, 60438 Frankfurt am Main, Germany \\ ${ }^{5}$ On leave from A.F. Ioffe Physical Technical Institute, 194021 St. Petersburg, Russian Federation
}

Received 4 March 2020

Published online 14 April 2020

(C) The Author(s) 2020. This article is published with open access at Springerlink.com

\begin{abstract}
The structure, formation and dynamics of both animate and inanimate matter on the nanoscale are a highly interdisciplinary field of rapidly emerging research engaging a broad community encompassing experimentalists, theorists, and technologists. It is relevant for a large variety of molecular and nanosystems of different origin and composition and concerns numerous phenomena originating from physics, chemistry, biology, or materials science. This Topical Issue presents a collection of original research papers devoted to different aspects of structure and dynamics on the nanoscale. Some of the contributions discuss specific applications of the research results in several modern technologies and in next generation medicine. Most of the works of this topical issue were reported at the Fifth International Conference on Dynamics of Systems on the Nanoscale (DySoN) - the premier forum for the presentation of cutting-edge research in this field that was held in Potsdam, Germany in October of 2018.
\end{abstract}

\section{Introduction}

This Topical Issue focuses on an interdisciplinary research field that gains more and more interest in the last few years and whose community grows continuously - the dynamics of systems on the nanoscale. The nanoscale dynamics determines a wealth of physical, chemical and biological processes and applications, such as the macroscale properties of materials, the catalytical activity of interfaces and the interaction of radiation with biological systems. However, its exploration and description requires unified experimental and theoretical approaches from different fields across the physical, chemical and biological disciplines.

Interatomic and intermolecular interactions determine the formation of nanoscale structures such as atomic and molecular clusters, nanoparticles and biomolecules, representing building blocks for further higher-order structures created by self-organization and self-assembly, and forming the basis of biomolecular and mesoscopic systems. Nanoscale structures possess unique and often size-dependent physical and chemical properties, which determine the functions of nanomaterials. The detailed

* Contribution to the Topical Issue "Dynamics of Systems on the Nanoscale (2018)", edited by Ilko Bald, Ilia A. Solov'yov, Nigel J. Mason and Andrey V. Solov'yov

a e-mail: ilia.solovyov@uni-oldenburg.de understanding and description of these structures, interactions, properties, functions and dynamics represent a veritable challenge for theoretical and experimental techniques, and recently the term Meso-Bio-Nano (MBN) Science [1] has been coined to describe the endeavor to establish new methods for the exploration of the dynamics of systems along various sizes and time scales.

A thorough understanding of MBN systems allows for an exploitation of novel phenomena on the nanoscale leading to an optimization of existing processes such as nanocatalysis $[2,3]$ or the exploration of novel applications e.g. involving radiation. The latter encompass nanofabrication technologies such as focused electron or ion beam induced deposition (FEBID or FIBID, respectively) [4], novel light source technologies [5], and novel radiotherapy treatments $[6,7]$.

In the meantime it became a tradition to discuss these topics at the International Conference "Dynamics of Systems on the Nanoscale" (DySoN), which started in Rome, Italy in $2010^{1}$. Subsequent events followed in 2012 in St. Petersburg, Russia (see Ref. [8] for the collection of contributions), in 2014 in Edinburgh, UK, and in October 2016 in Bad Ems, Germany ${ }^{2}$ (see also Ref. [9]). The most recent and fifth DySoN conference took place in October

\footnotetext{
1 www.dyson-conference.org

2 epjd.epj.org/component/toc/?task=topic\&id=772
} 
2018 in Potsdam, Germany ${ }^{1}$, bringing together a number of scientists from around the world ${ }^{3}$.

A broad range of scientific topical areas in the field has been discussed in Potsdam:

- The structure and dynamics of clusters, nanoparticles, biomolecules and bio-nano systems,

- Clustering and self-organization on the nanoscale,

- Cluster and biomolecular ensembles, complexes, nanostructured materials,

- Surfaces and interfaces,

- Nanoscale phase and morphological transitions,

- Reactivity and nanocatalysis,

- Irradiation driven transformations, fabrication and damage of MBN systems,

- Biomedical and technological applications of radiation,

- Thermal, optical and magnetic properties of $\mathrm{MBN}$ systems,

- Electron and spin transport in molecular systems,

- Collision and radiation processes, fusion, fission, fragmentation,

- Propagation of particles through media.

The next DySoN 2020 conference will take place in Santa Margherita Ligure, Italy on November 23-27, 2020.

In addition to the DySoN conference there are many other international meetings whose topics contribute to the knowledge about dynamics of systems on the nanoscale. Without pretending for the completeness let us mention here some of these conference series: The International Conference on Many-Particle Spectroscopy of Atoms, Molecules, Clusters and Surfaces ${ }^{4,5}[10]$, The Symposium on Size Selected Clusters (S3C) ${ }^{6}$, The Symposium on Atomic, Cluster and Surface Physics (SASP) ${ }^{7}$, and the meeting on Focused Electron Beam Induced Processing (FEBIP $)^{8}$.

In this Topical Issue on Dynamics of Systems on the Nanoscale recent developments and advancements of the field are presented, ranging from fundamental research on elementary atomic and molecular mechanisms to studies on a more applied level, and covering innovative theoretical and experimental techniques. A particular focus lies on clusters and nanoparticles, molecules and molecular systems, nanosystems, collision and radiation phenomena involving MBN systems, and application inspired case studies. Most of the contributions have been reported at the DySoN 2018 conference and in this Editorial, we present a brief overview of all the contributions.

\footnotetext{
3 epjd.epj.org/component/toc/?task=topic\&id=1068

4 epjd.epj.org/component/toc/?task=topic\&id=716

5 epjd.epj.org/component/toc/?task=topic\&id=1035

6 www.s3c.ch

7 sasp20.empa.ch

8 www.gla.ac.uk/schools/physics/febip2020
}

\section{Clusters and nanoparticles}

A group of atoms bound together by interatomic forces is called an atomic cluster [11]. There is no qualitative distinction between small atomic clusters and molecules. However, as the number of atoms in the system increases, atomic clusters acquire more and more specific properties making them unique physical objects different from both single molecules and the solid-state. The novelty of atomic cluster physics arises mostly from the fact that structural and dynamical properties of clusters explain the transition from single atoms or molecules to the solid-state [11]. Modern experimental techniques have made it possible to investigate this transition by, for example, studying the emergence of plasmon excitations, electron conduction band formation, superconductivity and superfluidity, phase transitions, and fission, in clusters of increasing size. Most of the aforementioned many-body phenomena exist in the solid-state but are absent for single atoms. The science of atomic clusters is a highly interdisciplinary field and concerns astrophysicists, atomic and molecular physicists, chemists, molecular biologists, solid-state physicists, nuclear physicists, plasma physicists, technologists, all of whom see them as a branch of their subjects. However, the physics of atomic clusters is a new subject in its right [11] and is an essential field of MBN Science [1].

In this Topical Issue Tarento presents a theoretical study on the ionization of small $\mathrm{Hg}, \mathrm{Cd}$ and $\mathrm{Zn}$ clusters and proposed a Hamiltonian for the calculation of the first and second ionization potentials [12]. The results are generally in agreement with experiments. Furthermore, the charge localization in the respective singly and doubly charged clusters was calculated and the stability of clusters discussed by the authors.

Precious metal nanoparticles such as gold and silver nanoparticles are frequently used for surface-enhanced Raman scattering (SERS), where the electric field enhancement upon excitation of the surface plasmon resonance is exploited [13]. Lackner et al. have presented an interesting way to synthesize Ag@Au core-shell nanoparticles in helium nanodroplets [14]. The advantage of this method is that the $\mathrm{Ag}: \mathrm{Au}$ ratio can be precisely controlled, which in turn allows the spectral position of the surfaceplasmon resonance to be turned. The authors have prepared particles with a diameter around $5 \mathrm{~nm}$ made of pure $\mathrm{Ag}$ or $\mathrm{Au}$ or with $\mathrm{Ag}: \mathrm{Au}$ ratios of 1:2, 1:1 and 2:1, respectively, thereby shifting the surface plasmon resonance between $447 \mathrm{~nm}$ (for bare $\mathrm{Ag}$ ) to $555 \mathrm{~nm}$ (for bare $\mathrm{Au})$. Finally, the SERS performance was studied using 4-methylbenzenethiol at three different excitation wavelengths $(473 \mathrm{~nm}, 532 \mathrm{~nm}, 633 \mathrm{~nm})$.

In another study SERS spectra of brominated nucleobases adsorbed on Ag nanoparticles are presented [15]. Excitation of the surface plasmon resonance not only leads to an enhancement of the electric field around the nanoparticles, but also to the formation of hot electrons, which can be transferred to the molecules. Dutta et al. have demonstrated hot-electron induced modification of brominated molecules by analyzing SERS spectra at different times of irradiation with an excitation laser at $532 \mathrm{~nm}$ and comparing the resultant spectra with those 
of non-brominated nucleobases. The results indicate that all the brominated nucleobases are subject to substantial hot-electron induced modification and that the cleavage of the $\mathrm{C}-\mathrm{Br}$ bond is a pivotal elementary step. It is likely that electrophilic molecules adsorbed onto plasmonic nanoparticles are in general subject to hot-electron induced reactions when irradiated with visible light matching the surface plasmon resonance of the particles. This could open up new strategies for cancer treatment using such plasmonic particles (i.e. photothermal therapy, PTT).

A theoretical study [16] investigated helium nanodroplets with impurities. Here the impurities were modeled through cesium atoms and the Coppens et al. studied the dynamic evolution of a Cs atom photo-excited from $6 s$ to $6 p$ and $7 s$ states on a helium droplet using timedependent DFT simulations. The authors concluded that, depending on the excited electronic state, the Cs impurity remains on the droplet surface or could be ejected. Furthermore it has been demonstrated that upon subsequent photo-ionization of the excited $\mathrm{Cs}$ atom the resulting $\mathrm{Cs}^{+}$ cation may either be ejected or come back to the droplet; this dynamical effect depends on the time delay between photo-excitation and photo-ionization. The authors have established the critical time delay separating these two different behaviors, as well as final ion velocities.

Hybrid $\mathrm{Au}_{38}\left(\mathrm{SCH}_{2} \mathrm{CH}_{2} \mathrm{Ph}\right)_{24}$ nanoclusters have been studied through DFT computations by Juarez-Mosqueda et al. [17]. Their study addressed an open question of the dynamics of these ultra-small metal systems, their decomposition mechanisms, and the energy that separates their structural isomers. Particularly, with DFT, the authors were able to compute the ground state energy and perform Born-Oppenheimer molecular dynamics of two $\mathrm{Au}_{38}\left(\mathrm{SCH}_{2} \mathrm{CH}_{2} \mathrm{Ph}\right)_{24}$ isomers. The energetic difference between the isomers has been established, and their decomposition mechanisms triggered by high temperatures investigated. The energy difference of the two isomers was established to be of the same order of magnitude as the energy difference between the fcc and hcp phases of bulk gold reported earlier. MD simulations revealed disordering and eventual fragmentation of the cluster structures at high temperature which seem to proceed via spontaneous formation of $\mathrm{Au}_{x}(\mathrm{SR})_{y}$ polymeric chains. These results greatly contribute to our understanding the possible decomposition mechanism, stability and robustness of existing and new monolayer-protected clusters.

Brabec et al. [18] examined theoretically how and to which extent physical processes can be retrieved from twocolor pump-probe experiments of atomic and molecular gases driven by an attosecond XUV pulse train and an infrared (IR) pulse. The He atom, the $\mathrm{N}_{2}$ molecule and $\mathrm{Na}$ clusters have been investigated with time-dependent density functional theory. The results are interpreted on the basis of a simple model system. Observables most commonly used in experiments have been analysed: ionization yield, photo-electron spectra, and angular distributions. It is reported that the basic time-dependent signatures are dominated by the interplay of IR laser and continuum electrons. System information, contained in the signal, in general requires careful disentangling from the effects of photon-electron dynamics.

\section{Molecules and molecular systems}

Another representative area of $\mathrm{MBN}$ science concerns investigations of structure and dynamics of molecules and molecular systems. Possible case studies may include proteins, DNA, lipid bilayers both in isolation and in assemblies. Interactions of these macromolecular systems with nanoparticles, various external environments, and radiosensitizing molecules are typically very complex and often poorly understood. Furthermore, investigation of the dynamics of molecular precursors for nanofabrication technologies, such as FEBID, FIBID, CVD, has recently defined as important in several modern technological applications, such as e.g., 3D nano-printing. In summary, molecular interactions, formation of complex molecular structures, developing materials with desired properties by their deposition on surfaces or their aggregation and transformations of these systems under different thermal, irradiation, biologically or technologically relevant conditions, or under various external stresses or external fields are the exemplar case studies in this research area.

In this Topical Issue this research area was represented by a theoretical contribution by Süß [19] focused on di- and triatomic platinum-containing molecules. They addressed the important question of the accuracy of different theoretical approaches that can be used to study small platinum-containing molecules and compared the computed bond lengths and bond dissociation energies with experimental data. Their paper contains a very systematic discussion of the various methods that include density functional theory based methods, including specifically 54 GGA, 17 meta-GGA, 36 hybrid, 103 mixed GGA/ meta GGA, 17 hybrid, 7 meta hybrid, 10 rangeseparated hybrid and 5 double hybrid density functionals. Furthermore, the performance of ab initio methods including Hartree-Fock, Møller-Plesset perturbation theory up to fourth order as well as coupled cluster theory up to perturbatively approximated triple excitations, i.e. $\operatorname{CCSD}(\mathrm{T})$, was investigated. The comparative analysis revealed that, the case of bond lengths, the smallest mean average deviation from experimental values yielding $0.3 \mathrm{pm}$ is found for the hybrid density functional TPSSh. Interestingly the investigation concluded that neither recent double hybrid functionals nor ab initio methods result in similar, commensurable accuracies. For the investigated bond dissociation energies the GGA functional TPSSVWN5 is closest to experiment with deviations of $6.97 \mathrm{kcal} / \mathrm{mol}$. Finally, the authors addressed various possible sources of errors that may explain the large mean average deviation from experiment in the case of $\operatorname{CCSD}(\mathrm{T})$, including the effect of basis set size, the influence of the multireference character of the molecular wave function, the quality of the HF determinant as reference wave function and the influence of core electron correlation.

\section{Nanosystems}

Nanostructured materials are materials with the characteristic size of structural elements of the order of or less 
than a few hundred nanometers, at least in one dimension. Examples include nanocrystalline materials, nanofibers, nanotubes, and nanoparticle reinforced nanocomposites. Atomistic modeling of nanosystems utilizes atoms as the elementary building blocks, providing the atomic resolution in the computational studies of materials' structure and properties. Such computational investigations combined with the rapid advances in the synthesis of nanostructured materials have enabled a new active area of materials research that strives to characterize and predict materials with enhanced or unique properties. Due to the nanoscopic size of the structural elements in nanosystems the interfacial regions start to play a dominant role in defining the physical properties of nanostructured materials. This implies that the behavior of nanomaterials cannot be understood and predicted by directly applying scaling arguments from the structure-property relationships developed for conventional polycrystalline, multiphase, and composite materials. New models are needed for an adequate description of the behavior and properties of nanomaterials.

In this Topical Issue nanosystems were investigated in several contributions. An investigation by Gula et al. [20] studied elastic properties of polystyrene. Here the authors employed a coarse-grained MARTINI force field and made some modifications to study polystyrene at the $\mu$ s timescale. The authors demonstrate that such extended simulations are necessary to take slow relaxation processes of polystyrene into account, and thereby compute reliably various elastic characteristics of polystyrene, such as the bulk modulus, the shear modulus, and the Poisson ratio. In their paper the authors use elastic properties to gauge a computational protocol for establishing various mechanical properties of a polymer system, based on the analysis of internal pressure in the system. They showed that the internal pressure of the system tends to saturate quickly enough such that $\mu$ s-long simulations are sufficient to predict elastic moduli close to those values reported in the experiment. Comparison with alternative computational techniques is extensively discussed and it is demonstrated that the suggested approach yields significantly more accurate results than the alternative analysis of internal energy of the system.

In another study Frederiksen and Solov'yov [21] investigated the structure and dynamics of isolated amino acid molecules deposited on top of a graphene surface. The study was inspired by recent investigations of cellular growth on specific functionalized substrates and the authors have made an attempt to describe how all twenty naturally-occurring amino acids interact with graphene; a study that could be potentially important to design special proteins that could improve cellular adhesion. Their investigations utilized classical molecular dynamics (MD) for amino acids in aqueous solution and in vacuo in tandem with quantum chemical calculations. The MD simulations were carried out for classical and polarizable CHARMM force fields. The simulations performed with the polarizable force field confirmed that adhesion of amino acids to the graphene surface may be significantly enhanced due to the polarization forces, which was further supported by quantum chemical calculations. The performed analysis revealed the role of polarization on amino acids' adhesion to the graphene surface.

Irradiation of nanosystems affects their structure and dynamics resulting in transformations and damage. A study by Valdes et al. [22] reports experimental and computational results of irradiation of multi-walled carbon nanotubes (MWCNT) deposited on a holey amorphous carbon (a-C) substrate with low energy $(2-10 \mathrm{keV} / \mathrm{u}) \mathrm{H}^{+}$ and $\mathrm{H}_{2}^{+}$molecular beams, impinging perpendicularly to the MWCNT axis. The energy distribution of protons traversing the nanotubes (either from the $\mathrm{H}^{+}$beam or dissociated from the $H_{2}^{+}$beam) was measured by the transmission technique in the forward direction. Two welldifferentiated peaks appear in the experimental energyloss distribution of the fragments dissociated from the molecular $\mathrm{H}_{2}^{+}$beam, in contrast to the ones detected with the proton beam. These peaks have been reproduced in computer simulations and their origin explained.

\section{Collision and radiation phenomena involving MBN systems}

Studies of collision and radiation phenomena involving MBN systems often provide valuable information about their structure, electronic properties, and interatomic interactions. Collision processes happen naturally in many different systems. They occur in gases and plasmas, the major properties of which to a large extent are determined by the physics of colliding particles, atoms, and molecules. They may happen on surfaces or inside liquids and solids. Collisions are traditionally used to investigate the unknown structure of various complex systems. At the time of Rutherford, the structure of atoms was understood through the famous collision experiments. Decades after the quark structure of nucleons was predicted it was validated through the experiments on electron and muon deep-inelastic scattering off protons and neutrons. In recent Free Electron Laser (FEL) experiments on the irradiation of proteins and clusters, the 3D atomistic structure of these complex systems is aimed to be photographed through X-ray diffraction of the FEL light on the atomic lattice of a single targeted molecular system [23]. Once achieved, this will provide a novel technology for the experimental recognition of the 3D structure of complex molecular systems, which is an essential task in numerous research and technological areas, such as biochemistry, pharmaceutics, nanofabrication, nanomaterials, and many others. In this section, we overview several case studies of collisions in different systems.

In one study by Surdutovich and Solov'yov [24] the possibility of multifragmentation of large Ar clusters by incident protons is assessed using the multiscale approach to radiation damage with ions. The characteristics of proton propagation in liquid Ar were calculated and the shock wave prediction is tested theoretically. The shock wave pressure was compared to the surface tension pressure at the surface of clusters and the condition for multifragmentation was explored. The dynamics of irradiated Ar clusters was studied using classical molecular dynamics 
simulations; different aspects of their survival or fragmentation are observed and discussed. These studies were performed for different values of impact parameter of ions with respect to spherical droplets. A size distribution of Ar droplets after irradiation was obtained.

In another investigation de Vera et al. [25] studied the fragmentation of a tungsten hexacarbonyl, $W(C O)_{6}$, molecule utilised in FEBID experiments by means of reactive molecular dynamics simulations. It was shown that distribution of the deposited energy over all degrees of freedom of the parent molecule leads to thermal evaporation of $\mathrm{CO}$ groups and the formation of $W(C O)^{+n}(n=0-5)$ fragments. $W C(C O)^{+n}(n=0-4)$ fragments are formed due to cleavage of a $\mathrm{CO}$ bond as a result of the localized energy deposition. Calculated fragment appearance energies are in good agreement with experimental data. These fragmentation mechanisms have a general physical nature and should take place in radiation-induced fragmentation of different molecular and biomolecular systems.

Villate et al. [26] studied application of radioactive gold nanoparticles for cancer treatment. It was demonstrated that dose enhancement by gold nanoparticles (AuNP) could increase the biological effectiveness of radiation damage in biomolecules and tissue. Their studies used a GEANT4 Monte-Carlo approach to characterise radioactive AuNP. The results specifically revealed an increase of the energy deposited in the vicinity of the AuNP up to $150 \mathrm{~nm}$, while this effect nearly vanishes for distances up to one micron. The authors demonstrated that the enhancement of the energy deposited increases with the relative gold mass percentage and can be adjusted by changing AuNP radius or clustering behaviour.

Study by Kowalska et al. [27] discussed the premature chromosome condensation (PCC) technique to study the initial DNA damage in order to distinguish between the biological and physical components of dose-effect curves. Specifically, G2-chromatid breaks in human lymphocytes were investigated at two different sampling times: directly after irradiation and 12 hours later. It was revealed that the G2-chromatid breaks follow linear-quadratic dependence for all studied cases and could be used to estimate the effective ion track radius. A study of the predicted physical comparison to the expected physical track radii allowed the authors to conclude on the importance of repair mechanism in damaged DNA.

\section{Application inspired case studies}

High energy radiation is used in many different applications, e.g., in the fabrication of nanostructures and cancer radiation therapy. The interaction of high energy radiation with condensed media results in the formation of secondary low-energy electrons, which can induce damage in biological environments of essential molecules such as the DNA. High energy radiation can have a potentially lethal effect on cells and can be used in biomedical applications, e.g., in ion-beam cancer therapy. The secondary electrons produced during the irradiation of nanostructured materials could also play a damaging role in nanotechnological applications, such as 3D nano-printing and FEBID. The action of secondary electrons can also lead to a significant reduction in material fabrication quality.

One relevant example is presented by Cipriani et al. [28], who studied the interaction of electrons with energies below $70 \mathrm{eV}$ with the $\mathrm{Ru}(\mathrm{CO})_{3} \mathrm{Br}$ complex. This compound is a potential precursor for FEBID, in which high-energy electron beams are used to fragment metalorganic compounds leading to three-dimensional deposits of pure metals. In order to improve the spatial resolution of the method reactions induced by secondary low-energy electrons are studied in order to minimize fragmentation. Cipriani et al. determined the apperance energies of the fragments generated by electron impacts on $\mathrm{Ru}(\mathrm{CO})_{3} \mathrm{Br}$ and compared these to calculated threshold energies using DFT and coupled cluster calculations. The mass spectra are dominated by sequential CO loss, allyl loss and concomittend allyl and CO loss. Calculation of the precursor ions provides detailed information on the individual reaction pathways and their study supports the picture that this class of compounds is not well-suited for FEBID.

Several studies are concerned with the interaction of radiation with fluorinated molecules, which are used as radiosensitizers in cancer radiation therapy. Among the most widely used therapeutics in the clinics are 5-fluorouracil (5FU) and gemcitabine, which are both highly reactive towards low-energy electrons [29]. Van der Burgt et al. [30] have studied the formation of positive ions from $5 \mathrm{FU}$ by electron impact from $0-100 \mathrm{eV}$ and by multiphoton ionization using laser thermal desorption. Appearance energies have been determined and some previously unknown fragmentation pathways identified and compared to the non-fluorinated uracil.

Gemcitabine is a therapetic based on cytidine, which is doubly fluorinated at the sugar moiety [31]. Huber et al. [32] have calculated the electron impact ionization cross sections of cytidine, singly and doubly fluorinated cytidine (i.e. Gemcitabine) and different variations of respective dimer sequences using the Deutsch-Märk and the binaryencounter-Bethe methods. The two methods agree well yielding maxima at $79-93 \mathrm{eV}$ with cross sectional values ranging from $27.97 \AA^{2}$ to $29.88 \AA^{2}$ for the single nucleosides. Interestingly the EI ionization cross sections of the nucleotide dimers could be approximated with an accuracy of about $6 \%$ by simply adding the cross sections of the individual building blocks. As a conclusion, the different molecules and sequences yield very similar EI ionization cross sections, but it was also found that a water environment can have a strong effect on the respective values.

Pavlov et al. [33] report systematic numerical analysis of the channeling properties for electrons and positrons in oriented straight and periodically bent diamond(110) crystals that can be utilised for the construction of novel crystal based light sources operating in the photon energy range $1-10 \mathrm{MeV}$, an energy range inaccessible to conventional synchrotrons, undulators and XFELs. The dependence of intensity of radiation emitted upon the projectile energy as well as on the bending amplitude was analysed using accurate numerical simulations of the channeling process. The simulation parameters, such as the crystal orientation, thickness and bending parameters of 
the crystals as well as the energy of the projectiles, were chosen to match those used in past and ongoing experiments. The peculiarities which appear in the radiation spectra are attributed to the interplay of various radiation mechanisms.

De Vera et al. [34] report the results of simulations of energy distributions for swift light ion beams after traversing cylindrical targets of different nature (liquid water and ethanol jets, as well as a solid aluminium wire). These simulations have been performed in order to reproduce a series of measurements intended to assess the stopping power of $0.3-2 \mathrm{MeV}$ ions. The authors discuss the relevance of the results obtained for the physical aspects of hadron therapy.

Brykalova et al. [35] advocate the utilisation of atomic and molecular physics methods in medical applications. In particular they address the question: how is the local electronic and atomic structure as well as molecular architecture of bone tissue distorted in case of skeletal pathology? X-ray diffraction and photoelectron spectroscopy techniques are applied to medial and lateral condyles of the femoral bone resected during total knee arthroplasty in patients with medial compartmental knee osteoarthritis (OA). The measured data are examined and discussed. It is shown that the skeletal pathology affects local electronic and atomic structure of mineral matrix in bone. For the first time site-dependent changes in crystallinity, binding energies and chemical composition of the intact and OA damaged areas of femur-saw-cuts are investigated. Specifically, in the sclerotic area crystallinity of the subchondral bone tissue increases, the binding energies of the $\mathrm{Ca}$ and $\mathrm{P}$ $2 p$ core levels demonstrate opposite shifts and the widths of the core $^{-1}$ photoelectron lines are maximized. These results can guide the development of novel approaches in medical imaging and diagnosis of bone tissue at the subcellular level.

\section{Concluding remarks}

To conclude this Editorial, let us mention a crucial issue that concerns the tools needed for a systematic approach to the field of research embraced by MBN Science. These tools, apart from theoretical models and experimental studies, also include computational aspects. MBN Science, as a research field, has only emerged recently from, primarily, atomic, molecular, and cluster physics together with the development of powerful computers and advanced computational techniques. The computational aspect of MBN Science provides the methodology for revealing novel features of structure and dynamics of nanoscopic and mesoscopic molecular systems. It also requires a high level of interdisciplinary collaboration since similar computational methodologies can be easily adapted to molecular systems of very different nature and origin.

To fully understand and exploit all the richness and complexity of the MBN-world, especially its all-atom dynamics, one needs to engage many disciplines ranging from physics and chemistry to materials and life science, exploiting technologies from software engineering and high-performance computing. This challenge led to the formulation and development of the multiscale software packages MBN Explorer [1,36] and MBN Studio [37]. These software packages have been designed to become powerful and universal instruments of computational research in the field of MBN Science, which should play a role of a "virtual microscope" and a "camera" capable to explore, simulate, record and visualize both structure and dynamics of the MBN-world with an atomistic level of accuracy, in order to both reproduce its known features and predict the new ones. In this Topical Issue, many contributions [12-22,24-35] provide case studies conducted with these packages and demonstrate their application to a broad variety of topical areas in MBN science as listed at the beginning of this Editorial. To be noted that these universal and powerful software packages are fully applicable to a broad variety of the topical areas of the MBN Science listed above in the introductory part of the Editorial.

Open access funding provided by Projekt DEAL. Financial support was provided by the University of Potsdam, University of Southern Denmark, and MBN Research Center. Further financial support provided by the German Research Foundation (DFG), Lundbeck Foundation, and H2020 project RISE-PEARL (reference number - 690991) is greatly acknowledged.

Open Access This is an open access article distributed under the terms of the Creative Commons Attribution License (https://creativecommons.org/licenses/by/4.0/), which permits unrestricted use, distribution, and reproduction in any medium, provided the original work is properly cited.

\section{References}

1. I.A. Solov'yov, A.V. Korol, A.V. Solov'yov. Multiscale modeling of complex molecular structure and dynamics with $M B N$ explorer (Springer, Springer International Publishing, 2017)

2. L. Liu, A. Corma, Chem. Rev. 118, 4981 (2018)

3. A. Gellé, T. Jin, L. de la Garza, G.D. Price, L.V. Besteiro, A. Moores, Chem. Rev. 120, 986 (2020)

4. I. Utke, A. Gölzhäuser, Angew. Chem. Int. Ed. 49, 9328 (2010)

5. W. Greiner, A.V. Korol, A.V. Solov'yov. Channeling and radiation in periodically bent crystals (Springer International Publishing, 2016)

6. A.V. Solov'yov, Nanoscale insights into ion-Beam cancer therapy (Springer International Publishing, 2017)

7. L. Sanche, Radiat. Phys. Chem. 128, 36 (2016)

8. A.V. Solov'yov, Dynamics of systems on the nanoscale, in Journal of Physics: Conference Series, 2nd International Conference (IOP Publishing, 2013), Vol. 438

9. A.V. Solov'yov, A.V. Korol (eds), European Physical Journal D, Topical issue "Dynamics of Systems at the Nanoscale" (Springer International Publishing, 2016)

10. A.N. Grum-Grzhimailo, Y. V. Popov, E.V. Gryzlova, A.V. Solov'yov, Eur. Phys. J. D 71, 201 (2017)

11. J.-P. Connerade, A.V. Solov'yov, W. Greiner, Europhys. News 33, 200 (2002)

12. R. Tarento, Eur. Phys. J. D 73, 260 (2019) 
13. R. Pilot, R. Signorini, C. Durante, L. Orian, M. Bhamidipati, L. Fabris, Biosensors 9, 57 (2019)

14. F. Lackner, A. Schiffmann, M. Lasserus, R. Messner, M. Schnedlitz, H. Fitzek, P. Pölt, D. Knez, G. Kothleitner, W.E. Ernst, Eur. Phys. J. D 73, 104 (2019)

15. A. Dutta, R. Schürmann, I. Bald, Eur. Phys. J. D 74, 19 (2020)

16. F. Coppens, J. von Vangerow, A. Leal, M. Barranco, N. Halberstadt, M. Mudrich, M. Pi, F. Stienkemeier, Eur. Phys. J. D 73, 94 (2019)

17. R. Juarez-Mosqueda, S. Malola, H. Häkkinen, Eur. Phys. J. D 73, 62 (2019)

18. T. Brabec, P.M. Dinh, C.Z. Gao, C.R. McDonald, P-G. Reinhard, E. Suraud, Eur. Phys. J. D 73, 212 (2019)

19. D. Süß, S.E. Huber, A. Mauracher, Eur. Phys. J. D, 73, 135 (2019)

20. Y.M. Beltukov, I. Gula, A.M. Samsonov, I.A. Solov'yov, Eur. Phys. J. D 73, 226 (2019)

21. A. Frederiksen, I.A. Solov'yov, Eur. Phys. J. D 74, 44 (2020)

22. J.E. Valdes, C. Celedon, M. Mery, J.D. Uribe, R. Segura, N.R. Arista, I. Abril, R. Garcia-Molina, Eur. Phys. J. D 73, 201 (2019)

23. B.W.J. McNeil, N.R. Thompson, Nat. Photonics 4, 814 (2010)

24. E. Surdutovich, A.V. Solov'yov, Eur. Phys. J. D 73, 241 (2019)
25. P. de Vera, Eur. Phys. J. D 73, 215 (2019)

26. Z. Villate, J. Mateo, M.B. Hahn, Eur. Phys. J. D 73, 95 (2019)

27. A. Kowalska, K. Czerski, E. Nasonova, P. Kutsalo, E. Krasavin, Eur. Phys. J. D 74, 17 (2020)

28. M. Cipriani, R.M. Thorman, C.R. Brewer, L. McElweeWhite, O. Ingólfsson, Eur. Phys. J. D 73, 227 (2019)

29. R. Schürmann, S. Vogel, K. Ebel, I. Bald, Chem. Eur. J. 24, 10271 (2018)

30. P.J.M. van der Burgt, M.A. Brown, J. Bockova, A. Rebelo, M. Ryszka, J.-C. Poully, S. Eden, Eur. Phys. J. D 73, 184 (2019)

31. J. Kopyra, A. Keller, I. Bald, RSC Adv. 4, 6825 (2014)

32. S.E. Huber, A. Mauracher, Eur. Phys. J. D 73, 137 (2019)

33. A.V. Pavlov, A.V. Korol, V.K. Ivanov, A.V. Solov'yov, Eur. Phys. J. D 74, 21 (2020)

34. P. de Vera, R. Garcia-Molina, I. Abril, Eur. Phys. J. D 73, 209 (2019)

35. X.O. Brykalova, N.N. Kornilov, A.A. Cherny, Y.A. Rykov, A.A. Pavlychev, Eur. Phys. J. D 73, 113 (2019)

36. I.A. Solov'yov, A.V. Yakubovich, P.V. Nikolaev, I. Volkovets, A.V. Solov'yov, J. Comput. Chem. 33, $2412(2012)$

37. G.B. Sushko, I.A. Solov'yov, A.V. Solov'yov, J. Mol. Graph Model 88, 247 (2019) 